\title{
Structural refinement of aqueous zirconium oxychloride using total scattering methods
}

\author{
Joe Andrew Rawlinson, Jennifer Elizabeth Readman
}

University of Central Lancashire, Preston, United Kingdom;

JRawlinson@uclan.ac.uk

Zirconium solutions have long been used as raw precursors in a range of applications to produce a diverse range of materials and products, including $\mathrm{ZrO}_{2}$ nanoparticles and high temperature ceramics which are widely used as catalysts and sorbents ${ }^{[1-3]}$. It is commonly accepted that the structure of the species in the zirconium solution is influential in the properties, chemical and physical, of any subsequent materials. It is for this reason that it is of critical importance that we fully understand the structure of the species within these solutions, and subsequently use this information to inform and adapt synthetic methods.

There is evidence that $\mathrm{Zr}(\mathrm{IV})$, in aqueous solution, has a tendency towards hydrolysis and polymerisation. A tetranuclear species $\left[\mathrm{Zr}_{4}(\mathrm{OH})_{8}\left(\mathrm{OH}_{2}\right)_{16}\right]^{8+}$ was proposed by Clearfield and Vaughn ${ }^{[4]}$. This species has a square $4-\mathrm{Zr}$ core held together by 8 hydroxyl bridges with 16 terminal waters. This structure was determined through the use of single crystal X-Ray diffraction (XRD) on solid samples of recrystallised $\mathrm{ZrOCl}_{2} \cdot 8 \mathrm{H}_{2} \mathrm{O}$ solution. Since its discovery in 1956, this structure has widely been regarded as being correct and its tetramer complex considered present in the aqueous phase ${ }^{[5]}$. Unfortunately, any attempts to study this species whilst still in aqueous solutions have been limited due to the lack of long range order in solution species, hence disallowing traditional laboratory crystallographic methods. However, recent developments of total scattering pair distribution function (PDF) have allowed for the study of disordered systems including glasses and, more importantly, liquids. This allows for the characterisation and modelling of solution species through methods similar to those traditionally associated with XRD and Rietveld refinements. Through the use of these methods we have been able to isolate contributions to the PDF pattern from the tetranuclear species, and subsequently refine the ideal solid state model to determine its nature in solution. Recent work by $\mathrm{Hu}$ et al. collected PDF data on aqueous zirconyl chloride and, through a method of fitting to Gaussians, confirmed that the general structure, proposed by Clearfield and Vaughn, is indeed present, but no refined inter atomic distances were obtained ${ }^{[6]}$. However through the use of structural refinements we have obtained a model which, whilst similar to the original Clearfield model, has some distortion with respect to bond lengths and angles in the terminal $\mathrm{H}_{2} \mathrm{O}$.

In this work we propose a refined version of the original model, first proposed by Clearfield. We also discuss the use of the Topas suite's rigid body editor and how, if a starting model is widely considered to be accurate, this offers enough flexibility to refine small differences to get a truly optimal model, with an extremely high level of precision, whilst ensuring that the statistics of the refinement are mathematically believable. The method used to obtain this refinement will be subsequently applied to further aqueous zirconium carboxylates which are yet to have their solution species identified.

[1] Geiculescu, A.C. et al., Sol-Gel Sci. and Tech., 2000. 17(1): p. 25-35.

[2] De Keukeleere, K., et al., Inorg. Chem., 2015. 54(7): p. 3469-3476.

[3] Feth, M.P., et al., Non-Crystalline Solids, 2005. 351(5): p. 432-443.

[4] Clearfield, A. et al., Acta Cryst., 1956. 9(7): p. 555-558.

[5] Hennig, C., et al., Inorg. Chem., 2017. 56(5): p. 2473-2480.

[6] Hu, Y.-J., et al., American Chem. Soc., 2013. 135(38): p. 14240-14248.

Keywords: Zirconium, Cluster, Solution Species, Liquid PDF 